Pbilosophia Philosophia Scientiæ

Scientie Travaux d'histoire et de philosophie des sciences

18-1|2014

Standards of Rigor in Mathematical Practice

\title{
Une nouvelle sémantique de l'itération modale
}

\section{Brice Halimi}

\section{OpenEdition}

\section{Journals}

Édition électronique

URL : http://journals.openedition.org/philosophiascientiae/923

DOI : 10.4000/philosophiascientiae.923

ISSN : 1775-4283

Éditeur

Éditions Kimé

Édition imprimée

Date de publication : 15 mars 2014

Pagination : 185-203

ISBN : 978-2-84174-665-1

ISSN : $1281-2463$

\section{Référence électronique}

Brice Halimi, « Une nouvelle sémantique de l'itération modale », Philosophia Scientiæ [En ligne], 18-1 |

2014, mis en ligne le 15 mars 2017, consulté le 03 novembre 2020. URL : http://

journals.openedition.org/philosophiascientiae/923 ; DOI : https://doi.org/10.4000/

philosophiascientiae.923 


\title{
Une nouvelle sémantique de l'itération modale
}

\author{
Brice Halimi \\ Université Paris Ouest (IREPH) \& SPHERE (France)
}

Résumé : Dire d'une proposition que, nécessairement, elle est nécessairement vraie, c'est affirmer incomparablement plus que ce que l'on affirme en disant simplement qu'elle est nécessairement vraie. C'est en effet, intuitivement, affirmer qu'elle est nécessaire quelle que puisse être la donnée de tous les mondes possibles à l'aune de laquelle sa nécessité est établie. C'est faire de cette donnée elle-même un possible parmi d'autres, et faire ainsi référence à des mondes possibles d'ordre supérieur. Cet article vise à formaliser la notion de monde possible d'ordre supérieur au moyen d'outils empruntés à la géométrie riemannienne. Le cadre sémantique proposé repose sur une collection ouverte de mondes possibles de niveaux croissants, par opposition à l'héritage leibnizien d'une totalité close de mondes possibles.

Abstract: Saying that a proposition is necessarily necessarily true claims incomparably more than simply saying that this proposition is necessarily true. Indeed, it amounts, intuitively, to saying that the proposition is necessarily true whatever the range of all the possible worlds may be. This range, upon which the assessment of simple necessity relies, then becomes a possible datum among others, which triggers the reference to higher-order possible worlds. This article aims at formalizing such a notion of high-order possible world, by using tools coming from Riemannian geometry. The semantical framework that is finally put forward involves an open-ended collection of possible worlds lying at higher and higher levels, in sharp contrast to the Leibnizian heritage of a fixed closed totality of possible worlds.

La sémantique moderne de la logique modale a repris de Leibniz la notion de monde possible, tout en cherchant à s'affranchir du cadre métaphysique dans lequel cette notion est ancrée chez Leibniz. Le terme de « monde possible» est, pour beaucoup d'auteurs, un simple moyen commode et imagé de désigner les unités d'interprétation des formules de la logique modale. On trouve néanmoins en logique modale un résidu essentiel de la métaphysique 
leibnizienne, dont même les auteurs les moins réalistes sont les héritiers : le fait que les mondes possibles forment un tout défini une fois pour toutes, dont la variation est hors de propos. Certes, à différentes interprétations d'un système de logique modale correspondront, dans la sémantique kripkéenne, différents ensembles de mondes possibles. Néanmoins, pour chaque interprétation donnée, l'ensemble de tous les mondes possibles est fixé et d'emblée clos. Or, si ce présupposé est naturel dans le contexte du système de Leibniz, il n'a pas de justification claire dans le contexte de la logique modale contemporaine, où les modalités itérées sont la règle.

En effet, Leibniz n'envisage à aucun moment de qualifier tel ou tel trait de notre monde de nécessairement contingent ou d'accidentellement nécessaire : une vérité est nécessaire ou contingente, mais dans l'un comme l'autre cas elle l'est absolument. Dans cette perspective, le fait que l'ensemble des mondes possibles forme une totalité close et absolue est dans l'ordre des choses. En revanche, les modalités itérées sont l'objet même des systèmes formels de logique modale, dans la mesure où la nécessité comme la possibilité y sont représentées par des opérateurs indéfiniment itérables. Or, dès lors que toute nécessité peut elle-même être posée comme contingente (à tort ou à raison, peu importe - seul importe que la contingence de la nécessité d'une vérité ait un sens), il devient naturel de considérer que l'ensemble des mondes possibles relativement auquel une vérité est dite nécessaire soit lui-même un ensemble contingent de mondes possibles, c'est-à-dire un ensemble de mondes possibles parmi d'autres ensembles possibles de mondes possibles. Et par là toute totalisation des mondes possibles est remise en cause, ou devrait du moins pouvoir l'être. Ce qui suit est l'exploration d'une telle voie.

\section{Les deux sens de la nécessité}

Par itération modale, il faut entendre toute superposition de qualifications modales. Dire, par exemple, d'une proposition qu'elle est «nécessairement nécessairement vraie», ou bien encore d'une proposition qu'elle est «nécessairement possiblement nécessairement vraie», c'est à chaque fois produire un cas d'itération modale. Le principe de l'itération modale remonte historiquement au moins à Clarence I. Lewis, c'est-à-dire à la tradition algébrique de la logique modale. En revanche, l'ouvrage de Rudolf Carnap intitulé Meaning and Necessity, qui a marqué un véritable renouveau de la logique modale, n'envisage à aucun moment la perspective d'une itération modale. Ceci s'explique par la conception que Carnap se fait de la nécessité comme validité logique. L'interprétation sémantique que Carnap propose de la nécessité renvoie en effet à l'espace logique de tous les mondes possibles définis comme ensembles maximalement consistants de propositions (pour un langage fixé); dans le cadre d'une telle analyse, une vérité peut bien être dite logiquement valide (vraie dans tous les mondes possibles), mais il est dénué de sens de se demander si 
cette validité logique est elle-même logiquement valide. Pour le dire dans les termes de Wittgenstein, l'énoncé qu'une proposition est possible ou nécessaire ne constitue pas lui-même l'énoncé d'une proposition ; il n'est donc, à proprement parler, ni vrai ni faux, et, a fortiori, ne saurait être dit possiblement ou nécessairement vrai : cf. [Wittgenstein 1922, 5.525, 6.124]. Cette idée, avec celle d'espace logique, a été reprise par Bas van Fraassen dans [van Fraassen 1969]. On peut dès lors distinguer deux grandes conceptions opposées de la nécessité : selon la première, toute qualification modale est fondamentalement itérable (et exprimée à cet effet par un opérateur du langage); selon la seconde, le redoublement de la nécessité (en particulier lorsque cette dernière exprime la notion métalinguistique de validité logique) n'a aucun sens ${ }^{1}$. S'il est tout à fait cohérent de combiner, comme Leibniz, une conception absolue des mondes possibles et un rejet des modalités itérées - la première n'étant que la traduction sémantique du second - il l'est moins, en revanche, d'autoriser des modalités itérées de degré arbitrairement grand tout en maintenant un ensemble absolu de mondes possibles.

La sémantique modale introduite par Saul Kripke en 1963 dans [Kripke 1963], fondée sur la considération de relations d'accessibilité entre mondes possibles, présente l'avantage d'une forme de conciliation de ces deux voies. Selon cette sémantique, un cadre pour la logique modale propositionnelle est une structure $\mathcal{F}=\left\langle W, R, w_{0}\right\rangle$ formée par un ensemble $W$ dont les éléments sont appelés par convention «mondes possibles», par un élément distingué $w_{0}$ de $W$ qui représente le « monde actuel», et par une relation binaire définie sur $W$, le sens intuitif de la condition $w R w^{\prime}$ étant que le monde $w^{\prime}$ représente une variante admissible de $w$ relativement au type de possibilité (physique, métaphysique, logique, etc.) en jeu. Pour un cadre donné, une valuation est une correspondance $V$ qui assigne, à chaque variable propositionnelle $p \mathrm{du}$ langage, une interprétation $V(p)$ consistant en un sous-ensemble de $W$ formé par tous les mondes possibles dans lesquels $p$ est réalisée. Un modèle de Kripke est un quadruplet $\mathcal{M}=\left\langle W, R, w_{0}, V\right\rangle$ obtenu à partir d'un cadre par la donnée supplémentaire d'une valuation $V$ sur les variables propositionnelles.

Tout système déductif pour la logique modale propositionnelle (dont on suppose ici la syntaxe connue) est caractérisé par un certain groupe d'axiomes. Un système est dit normal s'il inclut

1. À cet égard, une certaine interprétation de l'axiome 4, $\square p \rightarrow \square \square p$ (toute proposition n'est nécessaire qu'à la condition de l'être nécessairement), peut être considérée comme un croisement paradoxal des deux conceptions qu'on vient de distinguer. En effet, une justification souvent avancée de l'axiome 4 est la restitution de l'idée de validité logique. Mais l'interprétation de ' $\square$ ' comme exprimant la validité logique vaut pour une modalité simple $(\square \phi)$, non pour une modalité redoublée $(\square \square \phi)$. Par conséquent, de deux choses l'une : ou bien ' $\square$ ' est interprété par la validité logique, et dans ce cas l'axiome 4 est naturel mais informulable, car l'itération syntaxique de l'opérateur ' $\square$ ' n'a plus de sens clair ; ou bien l'itération modale est admise, mais dans ce cas l'interprétation de ' $\square$ ' par la validité logique, et avec elle la justification naturelle de l'axiome $\mathbf{4}$, devient problématique. 
- toutes les tautologies de la logique propositionnelle non modale : $(p \vee \neg p)$, $((p \rightarrow q) \rightarrow(\neg q \rightarrow \neg p))$, etc.

- l'axiome $\mathbf{K}: \square(p \rightarrow q) \rightarrow(\square p \rightarrow \square q)$,

et s'il a pour règles d'inférence

- le modus ponens

- la règle de nécessitation : si $\phi$ est un théorème, alors $\square \phi$ également (de sorte que toute tautologie est automatiquement comptée comme nécessaire)

- la règle de substitution uniforme : si une formule $\chi(p)$ contenant $p$ est un théorème, alors il en va de même de $\chi[\phi / p]$, pour toute formule $\phi$ du langage.

Le système qui résulte de ces axiomes et règles est appelé K. L'addition de l'axiome $\mathbf{T}, \square p \rightarrow p$ (ou, de façon équivalente, $p \rightarrow \diamond p$ ), définit le système KT. L'addition supplémentaire de l'axiome $4, \square p \rightarrow \square \square p$, définit le système $\mathrm{S} 4=\mathrm{KT} 4$; celle de l'axiome $\mathbf{5}, \diamond p \rightarrow \square \diamond p$, définit le système S5 = KT5.

La sémantique modale kripkéenne consiste dans les clauses inductives suivantes pour la satisfaction :

- $\mathcal{M}, w \vDash p$ ssi $w \in V(p)$;

- $\mathcal{M}, w \vDash \neg \phi$ ssi $\mathcal{M}, w \not \models \phi$;

- $\mathcal{M}, w \vDash(\phi \wedge \psi)$ ssi $\mathcal{M}, w \vDash \phi$ et $\mathcal{M}, w \vDash \psi$;

- $\mathcal{M}, w \vDash(\phi \vee \psi)$ ssi $\mathcal{M}, w \vDash \phi$ ou $\mathcal{M}, w \vDash \psi$;

- $\mathcal{M}, w \vDash \square \phi$ ssi, pour tout $w^{\prime}$ tel que $w R w^{\prime}, \mathcal{M}, w^{\prime} \vDash \phi$;

- $\mathcal{M}, w \vDash \diamond \phi$ ssi il existe $w^{\prime}$ tel que $w R w^{\prime}$ et $\mathcal{M}, w^{\prime} \vDash \phi$.

Une formule $\phi$ est dite valide dans $\mathcal{M}$ ssi $\mathcal{M}, w \vDash \phi$ pour tout $w \in W$. Elle est dite valide dans un cadre $\mathcal{F}$ ssi elle est valide dans tout modèle issu de $\mathcal{F}$. Enfin, elle est dite valide (tout court) si elle est valide dans tout cadre d'interprétation du langage.

Il est relativement aisé de voir qu'un cadre valide le système S4 (resp. S5) ssi sa relation d'accessibilité est réflexive et transitive (resp. une relation d'équivalence). La sémantique de Kripke est particulièrement propice à l'obtention de tels résultats, dits de complétude. Son avantage est également d'autoriser l'itération modale tout en la cantonnant à certaines limites, en la linéarisant. Ainsi, dans le cas de la nécessité redoublée :

$$
\mathcal{M}, w \vDash \square \square p \text { ssi } \forall v \text { tel que } w R v, \forall u \text { tel que } v R u, \mathcal{M}, u \vDash p .
$$

Comme on le voit, l'exploration du graphe défini par la relation d'accessibilité $R$ permet d'interpréter l'itération modale : plus haut est le degré modal d'une formule, plus longs sont les chemins à considérer sur le graphe. La sémantique kripkéenne traduit ainsi toute itération modale par une ramification. 
Elle constitue en cela une façon optimale d'interpréter l'itération modale au moyen d'un seul et même ensemble de mondes possibles $W$ explicitement donné dès le départ. Mais s'agit-il de l'unique façon d'interpréter l'itération modale? La suite de cet article voudrait suggérer une autre voie pour restituer toute la force de l'itération modale. Il s'agira moins, toutefois, de critiquer la sémantique de Kripke qu'au contraire de la prolonger et, en un certain sens qu'on précisera, de la généraliser.

Revenons au préalable sur la signification des modalités itérées. Admettons par exemple comme douée de sens l'assertion qu'une vérité nécessaire l'est pour des raisons contingentes. Un enchâssement de plans de validité est par là même suggéré : selon le plan le plus immédiat, la proposition considérée est tenue pour nécessairement vraie, mais, selon un plan plus profond qui relativise le premier, la nécessité attribuée à cette proposition apparaît elle-même comme un trait contingent. De même, permettre l'invocation de situations qui sont seulement possiblement possibles, c'est autoriser une marge de variation bien plus ample que celle représentée par le simple possible, puisque c'est accepter de faire varier le système de variation même sur lequel est fondée la notion de possible, et resituer ce système de possibilité comme étant seulement un parmi d'autres possibles, à l'intérieur d'un système de possibilité d'une échelle supérieure. Corrélativement, affirmer qu'une proposition est nécessairement nécessaire, c'est affirmer incomparablement plus que la simple nécessité de cette proposition; c'est en effet, intuitivement, affirmer qu'elle est nécessaire quelle que puisse être la donnée de tous les possibles.

La signification de l'itération modale demande donc d'introduire des contextes variables d'évaluation de ce qui est possible ou impossible. Comment le faire dans les termes de la sémantique des mondes possibles? Si la vérité d'une proposition donnée $p$ signifie sa satisfaction dans le monde actuel $w_{0}$, sa nécessité (c'est-à-dire la vérité de la proposition $\square p$ énonçant la nécessité de $p$ ) signifie sa satisfaction, non seulement dans le monde actuel, mais dans tout monde possible $w \in W$ (pour simplifier, on suppose ici que tout monde possible est accessible depuis $w_{0}$, ce qui revient à adopter l'acception la plus stricte possible de la nécessité). On passe ainsi de la considération d'un monde distingué $w_{0}$ à celle d'un ensemble de mondes $W$ dont $w_{0}$ n'est qu'un membre parmi d'autres possibles. De la même manière, affirmer qu'une proposition est nécessairement nécessaire devrait engager la considération d'un ensemble d'ensembles de mondes dont $W$ ne serait qu'un membre parmi d'autres possibles, et qui jouerait relativement à $W$ le rôle joué par $W$ relativement à $w_{0}$. Cet ensemble d'ensembles de mondes possibles devrait à son tour, dans le cas d'une nouvelle itération modale, jouer le rôle de monde actuel relativement à un ensemble d'ensembles d'ensembles de mondes possibles, etc. On appellera démultiplication modale chacune de ces étapes : le passage d'un monde à un ensemble de mondes de niveau supérieur, puis à un ensemble d'ensembles de mondes de niveau encore supérieur, et ainsi de suite.

Une telle progression n'est pas entièrement étrangère à la sémantique modale héritée de Kripke. En effet, l'itération syntaxique de l'opérateur de né- 
cessité induit, dans la sémantique kripkéenne, un changement dans le lieu d'évaluation des formules : $w_{0}$ pour les formules non modales, $E_{w_{0}}:=\{w \in$ $\left.W: w_{0} R w\right\}$ pour les formules de degré $1, \bigcup_{w \in E_{w_{0}}} E_{w}$ pour les formules de degré 2 (où $E_{w}:=\left\{w^{\prime} \in W: w R w^{\prime}\right\}$ ), et ainsi de suite. On aboutit donc bien à un emboîtement de systèmes de possibilité, au sens où chacun des mondes possibles $w$ appartenant à un certain système de possibilité $E_{w^{*}}\left(\right.$ si $\left.w^{*} R w\right)$ définit lui-même de son côté un nouveau système $E_{w}=\left\{w^{\prime} \in W: w R w^{\prime}\right\}$. En cela, la sémantique kripkéenne constitue une prise en compte véritable de l'itération modale.

On peut cependant rechercher une sémantique qui fasse davantage droit à la portée démultiplicative des modalités itérées. En effet, la sémantique kripkéenne confine d'avance tout ensemble de mondes à un sous-ensemble de $W$, ensemble clos, explicitement donné dès le début, de tous les mondes possibles en général. Certes, la collection de tous les mondes actuellement possibles est bien elle-même contrefactualisable - en ce sens que l'ensemble $E_{w_{0}}$ des mondes possibles relativement au monde actuel $w_{0}$ peut être remplacé par un autre ensemble $E_{w}$ - mais ce dans les limites d'un stock $W$ fixé. Ensuite, Kripke ne fait pas d'un monde possible un monde intrinsèquement relatif à un système de possibilité : le même monde $w$ peut en général être accessible à partir de mondes différents et se trouver au terme de branches de longueurs différentes. Par exemple, un même monde $w$ peut être 1-accessible à partir d'un certain monde $w_{1}$ (si $\left.w_{1} R w\right)$, mais seulement 3-accessible à partir d'un autre monde $w_{2}$ (si $w_{2} R w^{\prime}, w^{\prime} R w^{\prime \prime}$ et $w^{\prime \prime} R w$ pour un certain $w^{\prime}$ et un certain $w^{\prime \prime}$, sans que $w_{2} R w, w_{2} R w^{\prime \prime}$ ni $\left.w^{\prime} R w\right)$. Autrement dit, il est bien vrai que, dans la sémantique de Kripke, les possibilités varient d'un monde à un autre - certains mondes n'étant possibles (accessibles) que relativement à certains mondes; néanmoins, on ne peut pas distinguer de réels niveaux de possibilité : un monde possible, bien qu'accessible relativement à un autre monde, n'est pas en lui-même relatif à ce monde. Enfin, deux systèmes $E_{w}$ et $E_{w^{\prime}}$ n'ont en général rien à voir entre eux : s'il existe des relations entre les mondes, il n'en existe pas en général entre les ensembles de mondes, à l'échelle de ce qui serait un système de possibilité d'ordre supérieur. Sans doute, aucun des traits qu'on vient de souligner ne compromet la sémantique kripkéenne. Mais, précisément, l'objet de ce qui suit ne sera pas de critiquer cette dernière, il sera au contraire de radicaliser l'écart qu'elle marque avec la conception leibnizienne des mondes possibles, et également de généraliser d'une certaine manière le cadre qu'elle propose, d'un point de vue qui sera précisé à la fin.

Il ne s'agira pas non plus de contester tel ou tel axiome modal. Il faut en effet distinguer deux versants tout à fait indépendants l'un de l'autre : le choix d'un certain type de sémantique, et le choix d'un certain système axiomatique. Étant donné le premier, le second revient à sélectionner, parmi toutes les interprétations possibles, celles qui vérifient certains axiomes, mais précisément il ne change rien au principe général de la sémantique dans laquelle on décide de se placer, c'est-à-dire au sens donné aux opérateurs modaux en 
tant que tels, et par suite à l'itération modale. Le but du présent travail n'est donc pas de rejeter le système modal S5 correspondant à la conception leibnizienne de la nécessité. Le système S5 affirme que l'itération modale nous reconduit à l'éventail de possibles pris pour point de départ, que les possibilités actuelles épuisent d'emblée toutes les possibilités possibles. Il s'agit là d'une thèse qui ne va pas de soi. Mais cela signifie simplement que le système S5 correspond sémantiquement à une classe très particulière de structures au regard des structures d'interprétation en général.

Une dernière question préjudicielle reste à aborder. Elle concerne le concept même de monde possible d'ordre supérieur. Comment concevoir qu'un ensemble de mondes possibles puisse être considéré à son tour comme un monde actuel vis-à-vis d'un ensemble d'ensembles de mondes possibles? Comment un ensemble de mondes possibles pourrait-il lui-même constituer un monde possible de mondes possibles : comment plusieurs mondes possibles disjoints pourraient-ils former un monde, c'est-à-dire une totalité structurée et unifiée?

En réponse à cette difficulté, il convient de penser un monde possible d'ordre supérieur, non comme un ensemble de mondes possibles de niveau inférieur, mais à l'inverse comme un ensemble de mondes possibles de niveau supérieur, en faisant de chaque monde de niveau supérieur une certaine spécification du monde de niveau inférieur auquel il est attaché. Dans cette perspective, tout monde d'ordre supérieur émerge, non par synthèse, mais par analyse : tout monde de niveau inférieur devient un monde d'ordre supérieur du fait d'être regardé comme l'ensemble de toutes ses spécifications possibles au niveau supérieur - exactement comme l'analyse mathématique identifie une valeur $a$ à l'ensemble de tous les développements limités possibles d'une fonction ayant cette valeur; ou bien exactement comme la géométrie rapporte un point à l'ensemble des petites portions de courbes passant par ce point (autrement dit, à l'ensemble des façons de passer par ce point).

L'ordre d'un monde est donc à penser sur le modèle d'un ordre de dérivation. Selon ce modèle, tout monde possible recouvre un ensemble de mondes pour peu qu'il soit examiné de façon plus fine et apparaisse ainsi au carrefour de virtualités situées à un autre niveau, mais il ne cesse pas par ailleurs de constituer un monde, ce qui répond à la question de savoir comment une simple pluralité de mondes peut elle-même former un monde. Ce modèle présente en outre l'avantage technique qu'il est plus aisé d'analyser une structure interne en un monde que de construire une superstructure de mondes en l'absence de tout moyen canonique pour le faire.

Un monde de mondes possibles, ou monde possible d'ordre supérieur, n'est donc rien d'autre qu'un monde possible, c'est-à-dire un point d'un certain espace de possibilité, pris comme index d'un nouvel espace de possibilité, dont les points sont appelés mondes possibles de niveau supérieur. Parler d'un « monde de mondes possibles » n'est qu'une façon de désigner cette juxtaposition d'un monde possible et d'un système de mondes possibles relatifs à ce monde. Le problème n'est plus de faire en sorte qu'une pluralité de mondes possibles fasse 
monde, mais il reste à préciser à quelles conditions un espace suffisamment cohérent de mondes peut être introduit et attaché en propre à chaque monde possible pris comme point de départ. C'est ici que la géométrie intervient.

\section{Géométrisation}

\subsection{Démultiplication modale}

Dans tout ce qui suit, l'opérateur modal primitif sera, non la nécessité, mais la possibilité. La sémantique modale recherchée est une sémantique dans laquelle chaque introduction de l'opérateur ' $\diamond$ ' déplace l'évaluation des formules à un niveau supérieur au sein d'un étagement de systèmes de mondes possibles : partant des mondes possibles usuels de premier niveau, on doit ainsi aboutir à des mondes possibles de second niveau relatifs à tel ou tel monde de premier niveau, puis à des mondes possibles de troisième niveau dont chacun est à son tour relatif à un monde de deuxième niveau lui-même relatif à un monde de premier niveau, et ainsi de suite - le passage d'un monde $w$ à l'ensemble des mondes relatifs à $w$ constituant une opération de démultiplication indéfiniment itérable.

Dans cette représentation des choses, chaque monde donné est l'index du système des mondes possibles (de niveau supérieur) qui lui sont relatifs. Il est donc important d'avoir à l'esprit la distinction entre l'ordre et le niveau d'un monde possible. Un monde possible d'ordre 1 est un monde possible de niveau $n$ auquel est relatif un ensemble de mondes possibles de niveau $n+1$. Un monde possible d'ordre 2 est un monde de niveau $n$ auquel sont relatifs des mondes possibles de niveau $n+1$ eux-mêmes chacun d'ordre 1 . Et ainsi de suite. Comme on se ramène ultimement aux mondes de niveau 0 , les mondes possibles d'ordre $i$ (requis pour l'interprétation d'une formule modale de degré $i$ ), dorénavant, seront les mondes possibles de niveau 0 à chacun desquels est relative une succession de mondes possibles emboittés de niveaux respectifs $1,2, \ldots, i$ et d'ordres respectifs $i-1, i-2, \ldots, 0$. L'ordre correspond à la profondeur attachée aux mondes possibles de base, en fonction du degré modal de la formule à interpréter.

Une structure mathématique apte à servir de modèle doit donc schématiquement associer à chaque point d'un certain espace (point représentant un monde possible pris pour base) un nouvel espace qui représente le système des mondes relatifs à ce monde de base et dont chaque point pourra à son tour se voir associer un espace propre, et ainsi de suite. L'hypothèse directrice de tout ce qui suit est que l'espace tangent $T_{x} M$ à une variété différentiable $M$ en l'un de ses points $x$ répond à cette description, menant ainsi à une interprétation géométrique de la logique modale. (Une variété n'est rien d'autre que la généralisation mathématique de l'idée de surface, comme par exemple une sphère; 
l'espace tangent, la généralisation de l'image du plan tangent à une sphère en l'un de ses points.)

L'union disjointe $\coprod_{x \in M} T_{x} M$ de tous les espaces tangents à une variété $M$ donne elle-même lieu à une nouvelle variété, appelée le «fibré tangent » $T M$ de la variété initiale $M$, et accompagnée d'une projection naturelle $p: T M \rightarrow M$ définie par $p\left(v_{x}\right)=x$, pour tout vecteur $v_{x}$ tangent à $M$ en $x$. Comme le fibré tangent $T M$ est lui-même une variété, on peut en considérer le propre fibré tangent, $T T M=: T^{2} M$, puis à nouveau $T T T M=: T^{3} M$, etc., autrement dit $T^{i} M$ pour tout $i \geq 0$ (avec $T^{0} M=M$ et $T^{1} M=T M$ ). Pour une explication précise des notions géométriques mentionnées (variété différentiable, espace tangent, fibré tangent, etc.), voir [Lafontaine 1996, II, III].

Dans l'hypothèse qui est ici adoptée, une variété $M$ représente donc l'ensemble des mondes possibles de niveau $0 ; T_{x} M$, l'ensemble des mondes possibles de niveau 1 relatifs à $x \in M$, et $T M=\amalg_{x \in M} T_{x} M$, l'ensemble des mondes possibles de niveau 1 en général. Pris en soi, un monde possible $x \in M$ de niveau 0 est un monde possible au sens usuel; pris en tant que point de tangence entre $T_{x} M$ et $M$, ce même monde $x$ devient l'index d'un système de possibilité, un monde de mondes possibles. Pour un vecteur $v_{x}$ tangent à $M$ en $x, T_{\left(x, v_{x}\right)} T M$, représente l'ensemble des mondes possibles de niveau 2 relatifs à $\left(x, v_{x}\right) \in T M$, et $T T M$, l'ensemble des mondes possibles de niveau 2 en général; et ainsi de suite. Le niveau d'un monde possible $x$ peut donc être défini comme l'indice $i$ pour lequel $x \in T^{i} M$, et l'ordre de ce même monde comme la différence $j-i$, où $j$ représente le niveau maximum des mondes relatifs à $x$ qui sont considérés. Dans ce cadre, l'évaluation d'une formule non modale prend place en n'importe quel monde de niveau 0, c'est-à-dire en n'importe quel point de $M$; celle de $\diamond \phi$ fait appel à un ensemble d'ensembles de mondes possibles, à savoir l'ensemble $T M$ de tous les $T_{x} M(x \in M)$; de même, l'évaluation de $\diamond \diamond \phi$ met en jeu $T T M$; et ainsi de suite. Le passage de $M$ à $T M$, puis à $T T M$ correspond ainsi au passage de $\phi$ à $\diamond \phi$, puis à $\diamond \diamond \phi$. L'idée géométrique de démultiplication correspond au fait que la dimension de $T^{i+1} M$ est à chaque fois le double de celle de $T^{i} M$.

La nouvelle sémantique de l'itération modale qui est recherchée relève ainsi d'un projet de géométrie modale consistant finalement à comprendre toute itération modale selon un fibré géométrique. Dans une telle perspective, il est assez naturel d'interpréter chaque variable propositionnelle $p$ par un ensemble de courbes $^{2} \gamma: \mathbb{R} \rightarrow M$ sur $M$, puis de déclarer $p$ vraie en $x$ ssi l'une de ces courbes $\gamma$ passe par $x$. De même, on va le voir, la relation d'accessibilité

2. Une courbe sur une variété $M$ n'est rien d'autre qu'une fonction $\gamma: \mathbb{R} \rightarrow M$ supposée infiniment dérivable. La naturalité et l'importance des courbes sur une variété $M$ sont dues au fait que les seuls sous-ensembles naturels de $M$ sont les ensembles sous-jacents aux sous-variétés de $M$, et que, parmi ces sous-variétés, les courbes sont celles de plus petite dimension, et en cela les plus simples. Dans le contexte de la géométrie riemannienne, il est d'autant plus naturel de considérer les courbes d'une variété, que la considération des courbes, et notamment des géodésiques, est ce qui permet de définir la distance entre deux points. 
devient un ensemble de chemins continus et peut ainsi prendre tout son sens géométrique. Toutefois, l'idée d'une telle géométrie modale se heurte à un certain nombre de difficultés de construction, qu'il s'agit à présent d'analyser.

\subsection{Tâches}

Trois tâches sont en effet à prendre en considération. Il faut tout d'abord indiquer inductivement les moyens d'interpréter $\neg p,(p \wedge q)$ et $(p \vee q)$ par des ensembles de courbes respectifs, en partant des deux ensembles de courbes interprétant $p$ et $q$. Il faut ensuite pouvoir interpréter $\diamond p$ au moyen d'un ensemble de courbes se situant, non plus au niveau de $M$, mais au niveau de $T M$ : on peut parler à ce propos de relèvement modal. Il faut, en outre, pouvoir définir un relèvement d'un autre type pour n'importe quelle famille de courbes sur $M$. Considérons en effet une formule aussi simple que $(\diamond p \wedge q):$ si $\diamond p$ est interprétée par une famille de courbes sur $M$, l'ensemble des courbes sur $M$ interprétant $q$ doit pouvoir être transporté en un ensemble de courbes sur $T M$, de façon que l'interprétation de $q$ puisse être combinée à celle de $\diamond p$ - mais sans qu'un tel transport ne se confonde avec un relèvement modal, puisque, en l'occurrence, $\diamond q$ ne figure pas dans la formule considérée. On qualifiera donc ce transport de relèvement non modal.

Les tâches à remplir sont dès lors les suivantes. La première (T1) consiste à définir le relèvement modal de l'interprétation de n'importe quelle formule, faisant passer d'une famille de courbes sur $T^{i} M$ (interprétant une formule $\phi$ de degré modal $i$ ) à une famille de courbes sur $T^{i+1} M$ (interprétant la formule $\diamond \phi)$. La deuxième tâche (T2) est celle de définir le relèvement non modal de l'interprétation de n'importe quelle formule $\phi$. Du fait des écarts en degré modal entre sous-formules d'une même formule, toute famille de courbes sur $T^{i} M$ doit posséder un relevé non modal à $T^{i+1} M$. La troisième tâche (T3) est corrélative des deux premières : le relèvement (modal ou non modal) de n'importe quelle famille de courbes (interprétant une certaine formule) appelle la possibilité de relever n'importe quel monde possible. Supposons en effet que la variable $p$ soit interprétée par une famille de courbes sur une variété $M$. L'interprétation de $\diamond p$ sera alors une famille de courbes sur $T M$, et de même l'interprétation de $\diamond \diamond p$, une famille de courbes sur TTM. La formule $\diamond \diamond p$ sera vraie en $x \in M$ si l'une des courbes sur $T T M$ interprétant cette formule passe, non par $x$ (c'est impossible, puisque $x$ ne se situe pas sur TTM), mais par un certain élément de $T T M$ relié de manière précise à $x$. Cela suppose, de façon générale, qu'à chaque point $x_{i}$ de $T^{i} M$ corresponde un ensemble de points de $T^{i+1} M$, de sorte que tout point $x \in M$ donne finalement naissance à une suite $\pi^{0}(x)=\{x\}, \pi^{1}(x) \subseteq T M, \pi^{2}(x) \subseteq T T M, \ldots$ de contreparties de $x$. La dernière tâche (T4) a pour objet de vérifier que les axiomes élémentaires de toute logique modale normale sont vérifiés.

On aboutit ainsi à la définition suivante : un cadre modal géométrique consiste en une suite $\left(p_{i+1}: M_{i+1} \rightarrow M_{i}\right)_{i \geq 0}$ de fibrés sur la base d'une 


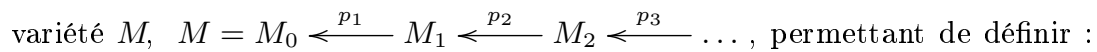
$1^{\circ}$ ) le relevé modal $\lambda(\Gamma)$ à $M_{i+1}$ de toute famille de courbes $\Gamma$ sur $M_{i}$; $2^{\circ}$ ) le relevé non modal $L(\Gamma)$ à $M_{i+1}$ de toute famille de courbes $\Gamma$ sur $\left.M_{i} ; 3^{\circ}\right)$ une suite $\left(\{x\}, \pi^{1}(x) \subseteq M_{1}, \pi^{2}(x) \subseteq M_{2}, \ldots\right)$ au-dessus de chaque point $x$ de $M$ vérifiant $p_{i+1}\left(\pi^{i+1}(x)\right) \subseteq \pi^{i}(x)$ pour tout $i \geq 0$. Une valuation sur un tel cadre est l'assignation, à chaque variable propositionnelle $p$, d'un ensemble de courbes sur $M$. Un cadre muni d'une valuation s'appelle un modèle modal géométrique.

\subsection{Modèles modaux métriques}

Le traitement des tâches (T1)-(T3) requiert l'ajout à $M$ d'une structure supplémentaire suffisamment riche pour permettre la définition relativement canonique des différents relèvements à opérer. Pour des raisons qui apparâ̂tront plus clairement au fur et à mesure, une voie assez naturelle consiste à se tourner vers la géométrie dite «riemannienne », qui représente le noyau de la géométrie différentielle moderne, et qui est une source très riche de constructions géométriques.

Le plan des développements qui vont suivre et mener jusqu'à la conclusion est ainsi le suivant. L'introduction d'un certain nombre de notions de géométrie riemannienne est tout d'abord nécessaire à la présentation du nouveau cadre sémantique qui vient d'être esquissé. Ce cadre, qui concrétise le projet de géométrie modale, sera ensuite défini en détail, compte tenu, notamment, des trois premières tâches qui ont été dégagées. Enfin, un certain nombre de premiers résultats seront présentés.

Une variété riemannienne est une variété munie d'une «métrique » $g$, qui rend possible de mesurer la longueur $g_{x}(v, v)$ de tout vecteur tangent $v \in T_{x} M$, autrement dit la vitesse de tout mouvement ayant lieu dans $M$, et par suite la distance entre deux points le long d'une courbe donnée sur $M$. La donnée d'une métrique $g$ sur $M$ induit la « connexion de Levi-Civita $\nabla$ associée à $g^{3}$. Intuitivement, l'opérateur $\nabla$ associe à deux champs de vecteurs ${ }^{4}$ $X$ et $Y$, définis au voisinage d'un point $x_{0}$ de $M$, la déviation infinitésimale $\left(\nabla_{X} Y\right)\left(x_{0}\right)$ de $Y$ en $x_{0}$ par rapport à la direction définie par $X_{x_{0}}$. Un champ de vecteurs $X$ le long d'une courbe $\gamma$ est dit parallèle si $\nabla_{X} X=0$ en chaque point de $\gamma$. Une courbe $\gamma$ dont le champ de vecteurs tangents $\gamma^{\prime}$ est parallèle s'appelle une géodésique : c'est une courbe dont le vecteur vitesse ne dévie jamais de lui-même. Dans le cas de l'espace euclidien, une géodésique n'est rien d'autre qu'une ligne droite. En général, c'est une courbe qui minimise localement la distance entre deux points par lesquels elle passe (ainsi des méridiens sur une sphère).

3. Cf. [Lehmann \& Sacré 1982, III] et [Gallot, Hulin et al. 2004, 2B] pour une définition de $\nabla$.

4. Un champ de vecteurs sur une variété $M$ est une application $X$ qui à tout point $x$ de $M$ associe un certain vecteur $X_{x}$ de $T_{x} M$. 
La connexion $\nabla$ associée à $g$ permet de définir le transport parallèle $J_{t, t^{\prime}}^{\gamma}(v)$ d'un vecteur $v \in T_{\gamma(t)} M$ le long d'une courbe $\gamma$, du point $\gamma(t)$ de paramètre $t$ au point $\gamma\left(t^{\prime}\right)$ de paramètre $t^{\prime}$. Intuitivement, $J_{t, t^{\prime}}^{\gamma}(v)$ est, comme son nom l'indique, le résultat du déplacement de $v$ le long de $\gamma\left(\right.$ de $\gamma(t)$ à $\left.\gamma\left(t^{\prime}\right)\right)$ lorsqu'on évite tout glissement comme tout pivotement, ce qui fournit le moyen de rapporter l'un à l'autre des vecteurs appartenant pourtant à des espaces tangents différents.

Par ailleurs, toute métrique $g$ supposée donnée sur une variété $M$ induit une métrique naturelle $g_{T}$ sur $T M$, appelée la métrique de Sasaki de $T M^{5}$. La construction de $g_{T}$ est itérable : en partant d'une variété riemannienne $(M, g)$, on définit une tour canonique de projections, à savoir $\left\langle p_{n+1}:\left(M_{n+1}, g_{n+1}\right) \rightarrow\right.$ $\left.\left(M_{n}, g_{n}\right)\right\rangle_{n \geq 0}$, avec $M_{0}=M, g_{0}=g$ et $M_{n+1}=T M_{n}, g_{n+1}=\left(g_{n}\right)_{T}$.

À présent, tout champ de vecteurs $X$ défini le long d'une courbe $\gamma$ sur $M$ constitue lui-même une courbe sur $T M$ définie au voisinage de $\gamma(0)=x$ : il suffit pour le voir d'écrire ' $X(t)$ ' au lieu de $X_{\gamma(t)}$, ce qui fait bien de $X$ une courbe $X:]-\epsilon, \epsilon[\rightarrow T M$ sur $T M$. Si le champ de vecteurs $X$ est parallèle le long de $\gamma, X^{\prime}(0) \in T_{X(0)} T M$, vecteur tangent à la courbe $X$, est dit horizontal. C'est notamment le cas si $\gamma$ est une géodésique et que $X=\gamma^{\prime}$. Une courbe $\delta: \mathbb{R} \rightarrow T M$ sur $T M$ est dite horizontale si $\delta^{\prime}(t)$ l'est pour tout $t \in \mathbb{R}$. L'ensemble des vecteurs horizontaux de $T T M$ forment un sous-fibré $H T M$ de $T T M$ tel que $H_{v_{x}} T M$ est isomorphe à $T_{x} M$ selon $T_{v_{x}} p_{1}$, et ce pour tout $v_{x} \in T_{x} M$. Par suite ${ }^{6}$, pour toute géodésique $\gamma$ de $(M, g)$ et tout vecteur $v \in T_{\gamma(0)} M$, il existe localement une unique courbe horizontale $\tilde{\gamma}^{v}$ sur $T M$ passant par $(\gamma(0), v)$ et telle que $p_{1} \circ \tilde{\gamma}^{v}=\gamma$. Cette courbe s'appelle «le relevé horizontal de $\gamma$ passant par $v$ en $t=0 »$ et est elle même une géodésique de $\left(T M, g^{T}\right)$. En particulier, pour toute géodésique $\gamma$ de $(M, g),\left(\gamma, \gamma^{\prime}\right)$ est le relevé horizontal de $\gamma$ passant par $\gamma^{\prime}(0)$, ainsi qu'une géodésique de $\left(T M, g^{T}\right)$.

Pour définir une structure de démultiplication conforme à la définition abstraite qu'on en a donnée, il convient d'introduire des mondes possibles de niveaux croissants, à chaque fois relatifs à un monde possible de niveau immédiatement inférieur. Pour cela, on suppose donnée une sélection $\mathcal{A}=$ $\left\{\gamma_{i}: i \in I\right\}$ de courbes sur $M$, appelées courbes d'accessibilité, qui représentent autant de chemins d'accessibilité entre mondes possibles. Ceci étant, on note $\bar{\gamma}$ l'ensemble $\{\gamma(t): t \in \mathbb{R}\}$ des points d'une courbe quelconque $\gamma$ et, pour $x \in \bar{\gamma}$, on note $t_{x}$ le paramètre correspondant à $x$ (autrement dit : $\left.\gamma\left(t_{x}\right)=x\right)^{7}$. Pour tout $x \in \overline{\gamma_{i}}, A^{1}(x):=\bigcup_{x \in \overline{\gamma_{i}}} \overline{\gamma_{i}}$ représente l'ensemble des mondes accessibles depuis $x$. Ces mondes, de niveau 0 , ne sont pas encore les contreparties de $x$ au niveau 1.

5. Cf. [Gallot, Hulin et al. 2004, 80].

6. Cf. [Gallot, Hulin et al. 2004, 100-102].

7. On supposera qu'aucune courbe d'accessibilité ne se coupe elle-même, et par suite que toute courbe d'accessibilité ne passe qu'une seule fois par chacun de ses points, ce qui permet de définir $t_{x}$ de façon univoque. 
Les contreparties de $x$ peuvent cependant se déduire des mondes accessibles depuis $x$, au moyen d'une application permettant de coder les points situés au voisinage d'un point donné $x$ d'une variété riemannienne $M$, par des vecteurs tangents de $T_{x} M$. Pour tout vecteur $v \in T_{x} M$, on peut en effet noter $c_{v}$ la courbe géodésique de $(M, g)$ telle que $c_{v}(0)=x$ et $c_{v}^{\prime}(0)=v^{8}$. L'application $v \in T_{x} M \mapsto c_{v}(1) \in M$ constitue un difféomorphisme entre un voisinage $V_{x}$ du vecteur nul $\overrightarrow{0}_{x}$ de $T_{x} M$ et un voisinage $U_{x}$ de $x$ dans $M$. Le plus grand réel $r_{x}>0$ tel que la boule $B_{x}:=B\left(\overrightarrow{0}_{x}, r_{x}\right)$ de centre $\overrightarrow{0}_{x}$ et de rayon $r_{x}$ soit incluse dans $V_{x}$ s'appelle le rayon de convexité de $M$ en $x$. Pour deux vecteurs quelconques $v$ et $w$ distincts dans $B_{x}$, on a donc toujours : $c_{v}(1) \neq c_{w}(1)$. On peut alors introduire l'ensemble $P^{1}(x):=\left\{v \in B_{x}: c_{v}(1) \in A^{1}(x)\right\}$ des représentants de $x$ dans $T_{x} M$, puis, au-delà de $T_{x} M$, à l'échelle de $T M$ en totalité, $\pi^{1}(x):=\left\{c_{v}^{\prime}(t): v \in P^{1}(x), t \in \mathbb{R}\right\}^{9}$. Le passage de $P^{1}(x)$ à $\pi^{1}(x)$ étend naturellement des mondes possibles (de niveau 1 ) isolés en des courbes de mondes possibles (de niveau 1). Les éléments de $\pi^{1}(x)$ seront appelés les contreparties de $x$ au niveau 1 . (Les mondes possibles de niveau 1 relatifs à $x$ seront, quant à eux, tous les éléments de $T_{x} M$, et par extension de $T M$, contenus dans le relèvement modal d'une courbe passant par $x$.)

Il est à présent nécessaire d'indiquer l'itération de la construction de $\pi=\pi^{1}$ le long de la tour de variétés riemanniennes de base $M$. Pour $n=0$, on pose simplement : $\pi^{0}(x)=\{x\}$. Comment définir $\pi^{2}(x)$ ? Toute courbe $\gamma_{i}$ sur $M$ définit un transport parallèle $J_{t, t^{\prime}}^{\gamma_{i}}: T_{\gamma_{i}(t)} \rightarrow T_{\gamma_{i}\left(t^{\prime}\right)}$ et par conséquent, pour tout $v \in T_{\gamma_{i}\left(t_{v}\right)} M$, une courbe $J_{v}^{\gamma_{i}}: t \mapsto J_{t_{v}, t}^{\gamma_{i}}(v)$ sur $T M$. On peut donc construire successivement $A^{2}(\alpha), P^{2}(\alpha)$ et $\pi^{2}(\alpha)$ pour $\alpha \in T M$ comme on a défini $A^{1}(x), P^{1}(x)$ et $\pi^{1}(x)$ pour $x \in M$, en remplaçant les géodésiques $c$ de $(M, g)$ par celles $c^{T}$ de $\left(T M, g_{T}\right)$, et les courbes de 1 -accessibilité $\gamma_{i}$ depuis $x \in M$ (pour un certain $x$ donné) par toutes les courbes de 2-accessibilité $J_{w}^{\gamma_{i}}$ depuis un élément quelconque $w \in T_{x_{0}} M$ de $\pi(x)$. Plus précisément, si l'on note $I_{0}$ l'ensemble des indices $j$ de $I$ tels que $x_{0} \in \overline{\gamma_{j}}$ (et $t_{j}$ le nombre tel que $x_{0}=$ $\gamma_{j}\left(t_{j}\right)$, pour chaque $j \in I_{0}$ ), l'ensemble des mondes possibles accessibles depuis $w$ est $A(w):=\bigcup_{j \in I_{0}} \overline{J_{t_{j},-}^{\gamma_{j}}(w)}$. L'ensemble des représentants de $w$ est alors $P(w):=\left\{u \in B_{w}: c_{u}^{T}(1) \in A(w)\right\}$ (en notant $c_{u}^{T}$ la géodésique de $\left(T M, g^{T}\right.$ ) telle que $c_{u}^{T}(0)=w$ et $\left.\left(c_{u}^{T}\right)^{\prime}(0)=u\right)$, et l'ensemble complet des contreparties de $w$ est $\pi(w):=\left\{\left(c_{u}^{T}\right)^{\prime}(t): u \in P(w), t \in \mathbb{R}\right\}$. L'ensemble des contreparties de $x$ au niveau 2 est l'ensemble des contreparties des contreparties de $x$ au niveau 1 , autrement dit $\pi^{2}(x):=\bigcup_{w \in \pi^{1}(x)} \pi(w)$. La définition de $\pi^{n}(x)$ pour $n \geq 3$ est analogue.

Il reste à définir les relevés respectivement modal et non modal de n'importe quelle courbe $\gamma$ de $M$ à $T M$, à $T T M$, et ainsi de suite. Pour cela, on introduit, pour chaque $n$, une sélection $\mathcal{C}_{n}$ de courbes sur $T^{n} M$, appelées courbes admissibles, qui sont les courbes dont l'interprétation d'une proposi-

8. L'existence et l'unicité de cette géodésique sont démontrables, voir [Gallot, Hulin et al. 2004, 81].

9. L'application $(v, t) \mapsto c_{v}^{\prime}(t)$ s'appelle le « flot géodésique » de la variété $(M, g)$. 
tion de degré modal $n$ est astreinte à se composer. On demande seulement que (i) si $\delta \in \mathcal{C}_{n+1}$, alors $p_{n+1}(\delta) \in \mathcal{C}_{n}$ et que (ii) pour toute $\gamma \in \mathcal{C}_{n}$, il existe $\delta \in \mathcal{C}_{n+1}$ relevant $\gamma$, c'est-à-dire telle que $p_{n+1}(\delta)=\gamma$.

Les relevés $\tilde{\gamma}^{\gamma^{\prime}(t)}$ sont les extensions naturelles à $T^{n+1} M$ d'une courbe $\gamma$ sur $T^{n} M$, et sont donc un choix naturel pour le relèvement modal de $\gamma^{10}$. Par ailleurs, comme le vecteur nul $\overrightarrow{0}_{x}$ est le représentant naturel de $x$ dans $T_{x} M$ $\left(\overrightarrow{0}_{x}\right.$ correspond visuellement au point de contact de l'espace tangent $T_{x} M$ avec $M)$, il est également naturel d'introduire, pour toute courbe $\gamma \operatorname{sur} T^{n} M$, la section nulle de $p_{n+1}$ le long de $\gamma$, autrement dit la courbe $\widehat{\gamma}$ sur $T^{n+1} M$ située au-dessus de $\gamma$ qui est donnée par : $\widehat{\gamma}(t)=\overrightarrow{0}_{\gamma(t)}$ pour tout $t \in \mathbb{R}$. On introduit alors, pour toute courbe $\gamma$ sur $T^{n} M$, les deux ensembles suivants de courbes sur $T^{n+1} M$ :

$$
\begin{aligned}
& \lambda(\gamma)=\left\{\tilde{\gamma}^{v} \in \mathcal{C}_{n+1}: \exists t, k \in \mathbb{R} \text { tels que } v=k \gamma^{\prime}(t)\right\} \\
& L_{n}^{n+1}(\gamma)=\{\widehat{\gamma}\} \cap \mathcal{C}_{n+1} .
\end{aligned}
$$

Comme l'écart en degré modal de deux sous-formules d'une même formule peut être strictement supérieur à 1 , on définit l'itération de l'opérateur de relèvement non modal : $L_{n}^{n}=\mathrm{id}, L_{n}^{n+m}=L_{n+m-1}^{n+m} \circ \ldots \circ L_{n}^{n+1}$.

On peut à présent définir un cadre modal géométrique $\underline{F}$ de type métrique comme un quadruplet $\left\langle M, g, \mathcal{A},\left(\mathcal{C}_{n}\right)_{n \geq 0}\right\rangle$. Une valuation $V$ dans ce cadre consiste en l'assignation, à toute variable propositionnelle $p$, d'un ensemble $V(p)$ de portions continues de membres de $\mathcal{C}_{0}{ }^{11}$. A toute courbe correspond l'ensemble $\bar{\gamma}$ de ses points, et pour tout ensemble $\Gamma$ de courbes on note : $\bar{\Gamma}=\bigcup_{\gamma \in \Gamma} \bar{\gamma}$, et par ailleurs $\lambda(\Gamma)=\bigcup_{\gamma \in \Gamma} \lambda(\gamma)$ et $L(\Gamma)=\bigcup_{\gamma \in \Gamma} L(\gamma)$. On suppose que chaque $V(p)$ est un ensemble clos par passage aux sous-courbes: toute courbe de $V(p)$, restreinte au voisinage de l'un de ses points, donne une courbe appartenant encore à $V(p)$. La conséquence de cette dernière hypothèse est que $\overline{V(p) \cap V(q)}=\overline{V(p)} \cap \overline{V(q)}$ et que $\overline{\mathcal{C}_{0} \backslash V(p)}=\overline{\mathcal{C}_{0}} \backslash \overline{V(p)}$. La valuation $V(\phi)$ de toute formule $\phi$ est alors définie par induction :

- $V(\neg \phi)=\mathcal{C}_{n} \backslash V(\phi)($ pour $\operatorname{deg}(\phi)=n)$;

- $V(\phi \wedge \psi)=V(\phi) \cap L_{m}^{n}(V(\psi))(\operatorname{pour} \operatorname{deg}(\phi)=n$ et $\operatorname{deg}(\psi)=m<n)$;

- $V(\phi \vee \psi)=V(\phi) \cup L_{m}^{n}(V(\psi))$ (sous la même hypothèse que ci-dessus);

- $V(\diamond \phi)=\lambda(V(\phi))$.

10. L'existence et l'unicité du relevé horizontal local de $\gamma$ ne sont assurées que si $\gamma$ est une géodésique de $\left(T^{n} M, g_{n}\right)$. C'est une raison pour limiter les sélections $\mathcal{C}_{n}$ à des ensembles de géodésiques.

11. Par « portion», il faut comprendre qu'une courbe de $V(p)$ peut ne décrire qu'une partie connexe de l'ensemble des points d'une courbe admissible, et avoir un vecteur vitesse variable (nul lorsqu'on atteint les extrémités de la courbe). On appellera «courbes admissibles », en un sens élargi, de telles portions de courbes admissibles au sens strict. 
Un modèle modal métrique $\underline{M}$ est un cadre modal métrique muni d'une valuation $V$.

Étant donné un tel modèle $\underline{M}$, on pose finalement, pour toute formule $\phi$ de degré modal $n$ et tout $x \in M$ :

$$
\underline{M}, x \vDash \phi \quad \text { ssi } \pi^{n}(x) \cap \overline{V(\phi)} \neq \emptyset .
$$

Une formule $\phi$ est donc vraie en $x$ si l'un des mondes possibles relatifs à $x$ contenus dans $V(\phi)$ coïncide avec une contrepartie de $x$ au niveau fixé par le degré modal de $\phi$.

On peut prendre un exemple simple pour illustrer les principales définitions : le plan euclidien $P^{2}$ muni d'un repère, avec pour courbes d'accessibilité toutes les droites horizontales (c'est-à-dire toutes les droites parallèles à l'axe des abscisses). Soit $O=(0,0)$ le point de $P^{2}$ pris pour origine. L'unique courbe d'accessibilité passant par $O$ est l'axe des abscisses : $A^{1}(O)=\{(a, 0): a \in \mathbb{R}\}$. On a par conséquent : $P^{1}(O)=\left\{\left((0,0),\left(\begin{array}{c}\alpha \\ 0\end{array}\right): \alpha \in \mathbb{R}\right\}\right.$, car un vecteur appliqué en $O$ ne peut avoir pour pointe un point de l'axe des abscisses qu'à la condition d'être horizontal. Par suite, $\pi^{1}(O)=\left\{\left((a, 0),\left(\begin{array}{c}\alpha \\ 0\end{array}\right)\right): a \in\right.$ $\mathbb{R}, \alpha \in \mathbb{R}\}$. Soit à présent $\gamma_{p}$ une courbe de $P^{2}$ faisant partie de la valuation de la formule $p$. Par définition, $\lambda\left(\gamma_{p}\right)$ est l'ensemble des courbes de la forme $t \mapsto\left(\gamma_{p}(t), k \gamma_{p}^{\prime}\left(t_{0}\right)\right)$ (pour un certain $k \in \mathbb{R}$ et un certain $t_{0} \in \mathbb{R}$ ). Pour que $P^{2}, O \vDash \diamond p$, il faut et il suffit qu'il existe une courbe $\gamma_{p}$ dans $V(p)$ vérifiant $\left(\gamma_{p}(t), k \gamma_{p}^{\prime}\left(t_{0}\right)\right)=\left((a, 0),\left(\begin{array}{c}\alpha \\ 0\end{array}\right)\right)$ (pour au moins un quintuplet $\left(t, k, t_{0}, a, \alpha\right)$ de nombres réels). Comme on peut toujours prendre $k=\alpha=0$, on obtient finalement :

$P^{2}, O \vDash \diamond p$ ssi il existe une courbe $\gamma_{p}$ dans $V(p)$ coupant l'axe des abscisses en au moins un point (qui peut être autre que $O$ ).

On retrouve ainsi une clause sémantique très proche des conditions de satisfaction de $\diamond p$ dans un modèle de Kripke : la sémantique modale géométrique fondée sur les modèles métriques permet de ressaisir la sémantique des modèles de Kripke comme une forme de cas particulier. La sémantique de Kripke correspond en effet au cas où l'espace topologique sous-jacent au graphe de la relation d'accessibilité est un espace discret. La sémantique fondée sur les modèles métriques en est ainsi à la fois une continuisation et une généralisation.

Un modèle modal métrique $\underline{M}$ donne en général à l'évaluation des formules un caractère essentiellement local. En particulier, la forme du codage de $A^{1}(x)$ par $P^{1}(x)$ dépend à chaque fois de la structure géodésique de $(M, g)$ au voisinage de $x \in M$, et il en va à nouveau de même aux niveaux supérieurs $\left(M_{n}, g_{n}\right)$ pour $n \geq 1$. L'esprit de la sémantique kripkéenne (de la clause donnée pour la satisfaction de $\diamond p)$ est donc conservé, mais mis en œuvre de 
façon à chaque fois propre au point considéré, ce qui est conforme à l'idée fondamentale que chaque monde, en cas d'itération modale, devient l'index d'un système de possibilité qui lui est propre.

L'axiome $\mathbf{T}, p \rightarrow \diamond p$, est bien valide. Supposons en effet (en se restreignant au cas où $p$ est une formule de degré modal nul) que $\pi^{1}(x) \cap \bar{\gamma} \neq \emptyset$, autrement dit que $\overrightarrow{0}_{\gamma\left(t_{x}\right)} \in \pi^{1}(x)$ pour une certaine courbe $\gamma \in V(p)$ et un

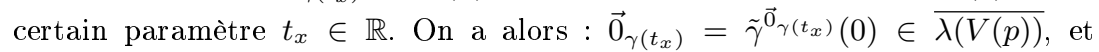
donc $\pi^{1}(x) \cap \overline{\lambda(V(p))} \neq \emptyset$. Par conséquent $\pi^{1}(x) \cap \overline{L(V(p))} \neq \emptyset$ implique $\pi^{1}(x) \cap \overline{\lambda(V(p))} \neq \emptyset$, et donc $\underline{M}, x \vDash p \rightarrow \diamond p$. De plus, la définition de $\square \phi$ entraîne qu'une courbe $\gamma\left(\operatorname{sur} T^{n+1} M\right.$ si $\phi$ est de degré $\left.n\right)$ appartient à $V(\square \phi)$ ssi $\gamma$ est disjointe de toute courbe ( $\left.\operatorname{sur} T^{n+1} M\right)$ relevant une courbe $\left(\operatorname{sur} T^{n} M\right)$ disjointe de $\overline{V(\phi)}$. On vérifie ainsi que l'axiome $\mathbf{K}, \square(p \rightarrow q) \rightarrow(\square p \rightarrow \square q)$, est également valide.

L'axiome 4 n'est généralement pas valide. En effet, la vérification de $\underline{M}, x \vDash$ $\diamond p$ suppose que $\lambda(V(p))$ passe par au moins un point de $\pi(x)$; en revanche, la vérification de $\underline{M}, x \vDash \diamond \diamond p$ suppose simplement que $\lambda(\lambda(V(p)))$ passe par au moins un point de $\pi(w)$ pour un élément $w$ de $\pi(x)$ qui peut tout à fait être autre que $x$. En revanche, on peut supposer que 4 devient valide si l'on se restreint à une classe très spéciale de cadres modaux métriques.

Supposons en effet que $(M, g)$ soit une variété riemannienne simple (c'està-dire telle que deux points quelconques de $M$ sont reliés par une unique géodésique), que $\mathcal{A}$ et $\mathcal{C}_{0}$ soient uniquement composés de géodésiques, que, pour tout $n \geq 0, \mathcal{C}_{n+1}$ soit l'ensemble des relevés horizontaux des courbes de $\mathcal{C}_{n}$, et enfin que deux courbes quelconques de $\mathcal{A}$ soient ou bien identiques à reparamétrisation près, ou bien disjointes. Un cadre modal métrique $\underline{F}$ vérifiant ces conditions sera dit minimal.

Proposition 1. L'axiome 4 est valide dans tout cadre métrique minimal.

Démonstration. Soit $\underline{F}=\left\langle M, g, \mathcal{A},\left(\mathcal{C}_{n}\right)_{n \geq 0}\right\rangle$ un cadre métrique minimal, qu'on suppose muni d'une valuation $V$. Pour toute géodésique $c$ de $M$, $\lambda(c) \subseteq \mathcal{C}_{1}$. En outre, $\tilde{c}^{k c^{\prime}(t)}=k c^{\prime}$, qui est une géodésique de $T M$. Par induction, on montre ainsi que, pour toute courbe $c$ de $\mathcal{C}_{0}$ et pour tout $n \geq 1$, $\lambda^{n}(c) \subseteq \mathcal{C}_{n}$ et que, pour tout $n \geq 0, \mathcal{C}_{n}$ se compose uniquement de géodésiques. Dans ce qui suit, pour plus de simplicité, on restreint l'axiome 4 à une variable propositionnelle $p$, mais la démonstration est analogue pour une formule $\phi$ quelconque, c'est-à-dire pour $\diamond \diamond \phi \rightarrow \diamond \phi$.

Pour $x \in M$, supposons qu'il existe une courbe $c_{x} \in \mathcal{A}$ passant par $x$. Cette courbe est par hypothèse une géodésique unique à reparamétrisation près. Tous les mondes accessibles depuis $x$ sont les points de $c_{x}$ et, pour tout point $y$ de $c_{x}$ distinct de $x, c_{x}$ est la seule géodésique reliant $x$ et $y$, car $(M, g)$ est supposée simple. On a donc : $P^{1}(x)=\left\{k_{0} c_{x}^{\prime}(0) \in B_{x}: k_{0} \in \mathbb{R}\right\}$ et $\pi^{1}(x)=\left\{k_{0} c_{x}^{\prime}\left(t_{0}\right): k_{0} c_{x}^{\prime}(0) \in B_{x}, k_{0}, t_{0} \in \mathbb{R}\right\}$. Par suite : 


$$
\begin{aligned}
& \pi^{2}(x) \\
= & \left\{\left(c_{u}^{T}\right)^{\prime}\left(t_{2}\right): c_{u}^{T}(1) \in \overline{J_{t_{0},-}^{c x}(w)} \text { avec } w=k_{0} c_{x}^{\prime}\left(t_{0}\right) \in B_{x}, u \in B_{w}, k_{0}, t_{0}, t_{2} \in \mathbb{R}\right\} \\
= & \left\{\left(c_{u}^{T}\right)^{\prime}\left(t_{2}\right): c_{u}^{T}(1) \in \overline{J_{t_{0},-}^{c}\left(k_{0} c_{x}^{\prime}\left(t_{0}\right)\right)} \text { avec } w=k_{0} c_{x}^{\prime}\left(t_{0}\right) \in B_{x}, u \in B_{w}, k_{0}, t_{0}, t_{2} \in \mathbb{R}\right\} \\
= & \left\{\left(c_{u}^{T}\right)^{\prime}\left(t_{2}\right): c_{u}^{T}(1) \in \overline{t \mapsto k_{0} c_{x}^{\prime}\left(t_{0}+t\right)} \text { avec } w=k_{0} c_{x}^{\prime}\left(t_{0}\right) \in B_{x}, u \in B_{w}, k_{0}, t_{0}, t_{2} \in \mathbb{R}\right\} \\
= & \left\{\left(c_{u}^{T}\right)^{\prime}\left(t_{2}\right): c_{u}^{T}(1)=k_{0} c_{x}^{\prime}\left(t_{1}\right) \text { avec } w=k_{0} c_{x}^{\prime}\left(t_{0}\right) \in B_{x}, u \in B_{w}, k_{0}, t_{0}, t_{1}, t_{2} \in \mathbb{R}\right\} .
\end{aligned}
$$

Mais, pour $k_{0}$ fixé, $c_{u}^{T}$ et $k_{0} c_{x}^{\prime}$ sont, à reparamétrisation près, deux courbes géodésiques de $T M$ ayant deux points d'intersection, puisque $c_{u}^{T}(0)=w=$ $k_{0} c_{x}^{\prime}\left(t_{0}\right)$ et que $c_{u}^{T}(1)=k_{0} c_{x}^{\prime}\left(t_{1}\right)$. Ces deux points sont bien distincts, car sinon on aurait $c_{\overrightarrow{0}_{w}}^{T}(1)=w=c_{u}^{T}(1)$, alors que $\overrightarrow{0}_{w} \in B_{w}$ et $u \in B_{w}$. On en déduit que $c_{u}^{T}=k_{0} c_{x}^{\prime}$ (à reparamétrisation près), et ainsi que $\pi^{2}(x)=\bigcup_{k_{0} \in \mathbb{R}} \overline{k_{0} c_{x}^{\prime \prime}}$. Si aucune géodésique de $\mathcal{A}$ ne passe par $x$, alors $P^{1}(x)=\pi^{1}(x)=\left\{\overrightarrow{0}_{x}\right\}$ et $\pi^{2}(x)=\left\{\overrightarrow{0}_{\overrightarrow{0}_{x}}\right\}$, donc l'égalité précédente reste valable : il suffit de prendre $k_{0}=0$. Si une (unique) géodésique de $\mathcal{A}$ passe par $x$ mais en se réduisant à $\{x\}$, alors $c_{x}^{\prime}=\overrightarrow{0}_{x}$ et $c_{x}^{\prime \prime}=\overrightarrow{0}_{\overrightarrow{0}_{x}}$, d'une part, d'autre part $\pi^{1}(x)=\left\{\overrightarrow{0}_{x}\right\}$ et $\pi^{2}(x)=\left\{\overrightarrow{0}_{\overrightarrow{0}_{x}}\right\}$, donc l'égalité est encore valable.

De plus, comme on l'a dit, pour $\gamma_{p} \in V(p), \lambda\left(\gamma_{p}\right)$ est composée (à reparamétrisation près) de toutes les courbes $k \gamma_{p}^{\prime}$, et toutes ces courbes sont des géodésiques contenues dans $\mathcal{C}_{1}$. De même, $\lambda\left(\lambda\left(\gamma_{p}\right)\right) \subseteq \mathcal{C}_{2}$ est composée (à reparamétrisation près) de toutes les courbes $k \gamma_{p}^{\prime \prime}$. Supposons à présent que $\langle\underline{F}, V\rangle, x \vDash \diamond \diamond p$, c'est-à-dire que $\pi^{2}(x) \cap \lambda(\lambda(V(p)) \neq \emptyset$. Cela signifie qu'il existe $\gamma_{p} \in V(p)$ et $k, k_{0}, t, t^{\prime} \in \mathbb{R}$ tels que $k_{0} c_{x}^{\prime \prime}(t)=k \gamma_{p}^{\prime \prime}\left(t^{\prime}\right)$. Il s'ensuit ${ }^{12}$ que $k_{0} c_{x}^{\prime}(t)=k \gamma_{p}^{\prime}\left(t^{\prime}\right)$, donc que $\overrightarrow{0}_{k_{0} c_{x}^{\prime}(t)}=\overrightarrow{0}_{k \gamma_{p}^{\prime}\left(t^{\prime}\right)} \in \overline{L(\lambda(V(p)))}$. En outre, $\overrightarrow{0}_{k_{0} c_{x}^{\prime}(t)}=\left(c_{\overrightarrow{0}_{k_{0} c_{x}^{\prime}(t)}^{T}}\right)^{\prime}(0)$ avec $c_{\overrightarrow{0}_{k_{0}} c_{x}^{\prime}(t)}^{T}(1)=k_{0} c_{x}^{\prime}(t)$, donc $\overrightarrow{0}_{k_{0} c_{x}^{\prime}(t)} \in \pi^{2}(x)$. Par conséquent $\pi^{2}(x) \cap \overline{L(\lambda(V(p)))} \neq \emptyset$. Ainsi, $\pi^{2}(x) \cap \overline{\lambda(\lambda(V(p)))} \neq \emptyset$ implique que $\pi^{2}(x) \cap \overline{L(\lambda(V(p)))} \neq \emptyset$, et donc $\langle\underline{F}, V\rangle \vDash \diamond \diamond p \rightarrow \diamond p$.

Soit $\mathrm{S} 4 *$ le système $\mathrm{S} 4$ privé de la règle de nécessitation, et dont la règle de substitution uniforme est restreinte au cas où le substituens possède un degré modal au plus égal à celui du substituendum. On peut montrer que le système S4* est complet relativement à la classe de tous les cadres métriques minimaux. La neutralisation de l'itération modale — neutralisation incarnée par l'axiome 4 - correspond ainsi, dans le cadre de la sémantique géométrique qui vient d'être proposée, à des modèles métriques très particuliers, ce qui est conforme au diagnostic que l'itération modale n'a pas un sens aisément neutralisable.

On pourrait objecter que la complexité de la sémantique fondée sur les cadres modaux géométriques rend cette dernière difficilement praticable d'un

12. En effet, pour toute courbe $\gamma$, le vecteur tangent $\gamma^{\prime}(t)$ est par définition attaché au point $\gamma(t)$ : si donc deux vecteurs tangents $\gamma_{1}^{\prime}(t)$ et $\gamma_{2}^{\prime}(u)$ sont identiques, ils appartiennent a fortiori au même espace tangent, ce qui implique que $\gamma_{1}(t)=\gamma_{2}(u)$. 
point de vue logique. Toutefois, ce serait adopter des critères techniques, et non philosophiques. Pour cette même raison, la facilité avec laquelle divers résultats de complétude peuvent être obtenus dans le cadre de la sémantique kripkéenne ne saurait être un argument. Seul importe ici le sens à donner à l'itération modale, et la possibilité d'exhiber une sémantique modale démultiplicative fidèle à ce sens. À cet égard, la sémantique géométrique qui vient d'être proposée est bien conforme à l'idée d'un déploiement progressif d'une hiérarchie ouverte de mondes possibles : elle introduit des mondes possibles d'ordres croissants au fur et à mesure que le degré modal de la formule considérée augmente.

\section{Conclusion}

La compréhension de l'itération modale défendue dans cet article repose sur l'idée suivante : à supposer qu'on raisonne en termes de mondes possibles pour analyser les notions modales aléthiques, la donnée de l'ensemble actuel des mondes possibles doit elle-même pouvoir être contrefactualisée, c'est-àdire replacée dans un ensemble d'ensembles de mondes possibles - monde de deuxième ordre lui-même contrefactualisable, et ainsi de suite. C'est cette structure indéfiniment ouverte du possible que vise à représenter la géométrie modale. Dire qu'il aurait été possible qu'un certain état de choses fût possible, c'est dire que cet état de choses aurait été possible si le système de possibilité défini par l'ensemble actuel des mondes possibles avait été différent.

Il ne va pas de soi de considérer l'itération modale comme douée de sens, et différents auteurs ont précisément refusé de le faire. Mais, dès lors que l'itération modale est acceptée, elle devrait être interprétée comme un saut radical, en rupture avec l'héritage leibnizien d'une totalité absolue de mondes possibles donnée une fois pour toutes. Passer d'une possibilité à une possibilité de possibilité (ou bien d'une nécessité à une nécessité nécessaire), ne devrait pas revenir simplement à augmenter d'un cran la longueur des explorations d'un même système de possibilité fixe, mais devrait au contraire impliquer une démultiplication, c'est-à-dire le passage d'un système de possibilité à un autre système de possibilité, au sein d'un système de possibilité de deuxième ordre. Telle a été la thèse conceptuelle défendue dans la première partie de cet article.

La seconde partie a consisté à proposer un cadre sémantique conforme à cette thèse. Il n'est pas seulement conceptuellement justifié, il est également techniquement possible de traduire l'itération modale par une démultiplication fondée sur une collection ouverte de mondes possibles articulés selon des niveaux indéfiniment croissants. La géométrie différentielle et la géométrie riemannienne fournissent des outils naturels au service d'une telle intuition, le passage d'une variété à son fibré tangent fournissant une image fidèle et intuitive de la démultiplication modale. D'autres raisons justifient de se tourner vers la géométrie : en particulier, la possibilité d'exploiter pleinement le sens 
géométrique de la notion d'accessibilité, et la possibilité de comparer un opérateur modal à un opérateur différentiel.

La sémantique géométrique proposée dans cet article est certes techniquement plus complexe que la sémantique kripkéenne, ce qui rend moins simple son emploi, et plus ardue l'obtention de théorèmes de complétude. Toutefois, un premier résultat a pu être obtenu, concernant l'axiome 4. En outre, le premier but de la géométrie modale est de faire droit à la signification démultiplicative des modalités. Il est de montrer précisément comment cela est possible, et par là, en retour, de rendre plus précise l'idée de démultiplication elle-même. Or l'interprétation de la logique modale fondée sur les modèles métriques suffit à ce propos, tout en ouvrant à une approche plus géométrique de la logique modale.

\section{Bibliographie}

Gallot, Sylvestre, Hulin, Dominique \& Lafontaine, Jacques [2004], Riemannian Geometry, Berlin : Springer.

Kripke, Saul [1963], Semantical considerations on modal logic, Acta Philosophica Fennica, 16, 83-94.

Lafontaine, Jacques [1996], Introduction aux variétés différentielles, Grenoble : Presses Universitaires de Grenoble.

Lehmann, Daniel \& Sacré, Carlos [1982], Géométrie différentielle des courbes et des surfaces, Paris : PUF.

van Fraassen, Bas [1969], Meaning relations and modalities, Noûs, 3(2), $155-167$.

Wittgenstein, Ludwig [1922], Tractatus Logico-Philosophicus, London : Routledge. 